\title{
TRIPLEX FORMING OLIGONUCLEOTIDES - TOOL FOR GENE TARGETING
}

\begin{abstract}
Martin Mojžžšek
Charles University in Prague, Faculty of Medicine in Hradec Králové, Czech Republic: Department of Medical Biology and Genetics

Summary: This review deals with the antigene strategy whereby an oligonucleotide binds to the major or minor groove of double helical DNA where it forms a local triple helix. Preoccupation of this article is triplex-forming oligonucleotides (TFO). These are short, synthetic single-stranded DNAs that recognize polypurine:polypyrimidine regions in double stranded DNA in a sequence-specific manner and form triplex. Therefore, the mechanisms for DNA recognition by triple helix formation are discussed, together with main characteristics of TFO and also major obstacles that remain to be overcome are highlighted. TFOs can selectively inhibit gene expression at the transcriptional level or repair genetic defect by direct genome modification in human cells. These qualities makes TFO potentially powerful therapeutic tool for gene repair and/or expression regulation.
\end{abstract}

Key words: Triple helix; Triplex forming oligonucleotides; Gene expression

\section{Introduction}

The biological role and function of DNA triple-helices has been a mystery since their discovery in the late 1950's (22). After numerous experiments in vitro and years of research, the triplex-mediated inhibition of transcription was demonstrated for the first time in the 1988 (8). Since that time, many projects were done with high optimism and effort, and triplex-based therapeutics (triplex forming oligonucleotides, TFO) have made significant progress in the last decade. However, to realize fully the potential of the gene therapy set of immediate problems must be overcome: (i) TFO uptake into cells, (ii) TFO stability in vivo must be improved, (iii) related binding sites must be evaluated, (iv) the restricted binding code must be expanded. The main goal of this article is to summarize current knowledge about biological properties of TFO, its biological activity, obstacles in wide gene therapy use and selected ways to enhance TFO qualities.

\section{Regulation of gene expression by DNA targeting}

Successful gene-targeting reagents must be functional under physiological conditions and must bind chromosomal target sequences embedded in chromatin. Triplex-forming oligonucleotides (TFOs) recognize and bind specific sequences via the major groove of duplex DNA, can be en- hanced in many aspects and have potential for gene targeting in vivo.

Approaches for regulation of gene expression based on sequence-specific targeting of nucleic acids by oligonucleotides take advantage of hydrogen bonds that form between the bases of dsDNA and oligonucleotide. Hydrogen bonds are relatively weak interaction, and stability of the structure formed depends upon the sequential binding of bases. There are two possible ways how to use oligonucleotides as inhibitors of gene expression, they are called antigene and antisense approach.

Target for antisense strategy is a ribonucleic acid (RNA), where translation of RNA is affected by several different mechanisms such as: (i) translational arrest by binding to the translational initiation codon, (ii) disruption or immobilization of ribosomal complex and (iii) cleavage of mRNA by RNase $\mathrm{H}$.

Antisense strategy is not covered by this article, for detailed view on antisense technology see review of Braasch DA and Corey DR (7).

In the antigene approach, oligonucleotides are synthesized that are complementary to the DNA sequence. Within the frame of antigene approach are at least three strategies under active study: minor groove binding polyamides, strand displacing PNAs (peptide nucleic acid oligomers), and binding of major groove by triplex forming oligonucleotides (TFO). 


\section{Polyamides}

Polyamides are a class of heterocyclic small molecules with the potential of controlling gene expression by binding to the minor groove of DNA in a sequence-specific manner, and usually refer to polymers containing N-methylimidazole and N-methylpyrrole amino acids. Crescent-shaped polyamides bind as antiparallel dimers with each polyamide making specific contacts with each strand on the floor of the minor groove. An Im/Py pair distinguishes $\mathrm{G} x \mathrm{C}$ from $\mathrm{C} \times \mathrm{G}$ and both of these from $\mathrm{A} \times \mathrm{T} / \mathrm{T} \times \mathrm{A}$ base pairs. A Py/Py pair specifies A,T from G,C but does not distinguish AT from T x A. To break this degeneracy White et al (64) added a new aromatic amino acid, 3-hydroxypyrrole (Hp), to increase the repertoire to test for pairings that discriminate $\mathrm{A} \times \mathrm{T}$ from $\mathrm{T} \times \mathrm{A}$. They found that replacement of a single hydrogen atom with a hydroxy group in an $\mathrm{Hp} / \mathrm{Py}$ pairing regulates affinity and specificity by an order of magnitude and therefore able to distinguish all four Watson-Crick base pairs in the minor groove of DNA (64). See papers for review of polyamides $(13,63,64)$.

\section{PNAs}

PNAs (peptide nucleic acid oligomers) are artificially constructed DNA analogs, where (2-aminoethyl) glycine carbonyl unit linked to the nucleotide bases through the glycine amino nitrogen and methylene carbonyl linkers has replaced normal sugar-phosphate backbone.

As a result, PNAs have neutral backbone and hybridization is not affected by intrastrand repulsion and occurs with enhanced affinity (18) and rates of association (50). PNAs seems to do not be substrates for nucleases or proteases (12). Under many condition, compared to DNA and RNA, PNAs have improved flexibility and PNA:DNA duplex is more stable than DNA:DNA complex (18). PNA can inhibit translation of target genes by hybridization to mRNA, using a steric blocking mechanism, or by strand invasion within relaxed DNA, by formation four-stranded complex $(33,41)$. Reviews of PNAs can be seen in papers of Nielsen PE or Gambari R $(24,40)$.

\section{Triplex forming oligonucleotides}

This approach utilizes the ability of single-strand nucleic acid to bind major groove of double helix DNA by Hoogsteen hydrogen bonds in sequence-specific manner and form triple-helix structure $(22,23,29)$. To form triple helix is required a polypurine:polypyrimidine tract within the target duplex, which are common in mammalian genomes (4). Typical TFO is from $15 \mathrm{bp}$ to $25 \mathrm{bp}$ long. TFO can be composed from pyrimidine motif or purine-rich sequence.

Pyrimidine TFO binds to purine strand of DNA duplex in parallel orientation (based on the triplets $\mathrm{C}^{+}(\mathrm{GC})$ and $\mathrm{T}(\mathrm{AT})$ ). $\mathrm{C}^{+}$means that cytosine is protonated. Protonation is required for triplex formation and arise at acidic $\mathrm{pH}$
(4.5-6), and therefore limits in vivo usability. Purine-rich TFO binds to the purine strand of the duplex in antiparallel orientation (triplex is build up from triplets of the $\mathrm{G}(\mathrm{GC})$ and A(AT) types). Several studies have been done to improve TFO activity under physiological conditions by oligonucleotides modification; it is discuss later on in this paper.

Following chapters are focused on TFO; properties of PNAs are reviewed in papers of Nielsen PE or Gambari. It remains to be seen if polyamides approach will be developed for more widespread in vivo work, and therefore it is not discuss more deeply.

\section{Biological roles of TFO}

In early biological studies, most envisioned potential applications were gene expression inhibition by blocking of transcription initiation or elongation at a DNA duplex target, disruption of necessary DNA structure or blocking of regulatory sites $(16,25,28,44)$. Transcription has been shown to be blocked at region where triplex is formed by prevention of binding transcription machinery factors $(17,36)$; and several studies have demonstrated that TFOs can decrease gene expression in mammalian cells in a directed way (47).

Alternative strategy use TFO to mediate genome modification, resulting in a change in target sequence $(55,59)$. The changes of target sequence can be permanent, and therefore this strategy has a potential as a tool for gene knockout and/or correction. DNA damaging agents have been successfully coupled to TFOs to induce site-directed DNA damages. Very often are used TFO linked to alkylating compounds, 125I-labeled TFO, TFO attached to conversion-electron-emitter, TFO with photoactivatable agent $(27,42,58)$. Photoactivable molecules attached to TFO provide the advantage of „switching on" when irradiated with a light of appropriate wavelength. Most of the photoactivable molecules work optimally when irradiated by wavelengths $<700 \mathrm{~nm}$, very often is used psoralen and UV radiation $(3,15,26,28,57)$. However, for use in biological environment the red-shifted wavelengths activable molecules are preferred since the bodily tissues are most transparent in these spectral regions.

\section{TFO induced mutations and recombinations}

It would be mistake to conclude from previous data that only mutagen-conjugated TFOs are capable to induce mutations in the target sequence. Mutagenicity of unconjugated TFOs was shown to be a consequence of the stimulation of DNA repair by the formation of the triple helix, which seems to be recognized by the nucleotide excision repair complex as a lesion (62). Moreover, TFOs (without any DNA reactive agents) administered for several constitutive days can induce mutations at specific genomic site in the somatic cells of adult mice (56). In this study, mice treated with the sequence-specific TFO had a fivefold elevated mu- 
tation rate in the target gene. All tissues tested showed TFO-induced mutagenesis except the brain, consistently with TFOs inability to cross blood-brain barrier.

Another application of the triple helix formation is triplex-induced recombination. Both conjugated-TFOs and unconjugated-TFOs could trigger recombination $(20,21)$. The frequency of the recombination depends heavily on the chosen method for the delivery of the TFO into the nucleus, and varies from sevenfold (for cationic liposomes) to more than 1000-fold (microinjection into the nucleus) increases of recombinants over the background (34).

As mentioned above, the third strand of nucleic acid has the ability to provoke DNA repair and stimulate recombination. TFO linked to a short DNA fragment homologous to the target site (except for the base pair to be corrected) can be used to mediate targeted gene conversion (31). In this bifunctional molecule, the TFO domain mediates site-specific binding to target the molecule to the desired gene. This binding also triggers repair to sensitize the target site to recombination. The tethered homologous donor fragment can participate in recombination and gene conversion with the target gene to correct or alter the nucleotide sequence. Chan, P.P. and his colleagues (31) used this approach in vitro successfully to correct single base-pair mutation, correction frequencies were in the range of $0.1-0.5 \%$. Both parts of such a bifunctional molecules can be linked to each other either by covalent linkage $(5,11)$ or by annealing via a short stretch of complementary nucleotides (5).

\section{Enhancement of the triplex binding code}

The requirement of long (>15 bp) polypurine: polypyrimidine sequence to form triplex is assurance of high specificity, but at the same time, it becomes the limitation of this approach. Often polypurine:polypyrimidine site is interrupted by inverted nucleotides, or do not span or overlap the transcription-binding site. It was shown that even single mismatch at the center of the triplex destabilize the triple helix (37). Recently some progress in the case of interrupted target polypurine:polypyrimidine sequence by one or two inverted nucleotide(s) (purine in the stretch of pyrimidines or pyrimidine in the stretch of purines) was made with TFOs modified at the sites of inversions. Generally, the major reasons for instability of triplexes on mixed sequences are possible hydrogen-bonding patterns. Purine bases engaged in Watson Crick pairing can form two additional hydrogen bonds, while duplex pyrimidines can only form one additional hydrogen bond. There are several categories of compounds challenging for efficient binding at inversion site: natural bases, intercalators, analogues that bind inverted pyrimidine by single hydrogen bond and analogues that bind inverted purine by two hydrogen bonds. Although natural bases in parallel triplexes form triplets at inverted base pairs, these triplexes are less stable then perfectly matching triplexes (61). Intercalators are used to stabilize the triplex, usually are incorporated into the TFOs adjacent to the mismatch site. Unfortunately, stability enhancement is at the cost of some degree of sequence specificity. Analogues that bind $\mathrm{C}: \mathrm{G}$ interruption both in pyrimidine and purine motif triplexes have been designed. Some of them have enhanced specificity because of the interaction with both cytosine and the guanine in the inverted base pair (30). To overcome some of the steric problems associated with base pair inversions, Durland et al. (14) substituted several natural bases of triplex by azoles derivates, that have smaller aromatic ring.

The formation of triple helical structures by association of a double helix containing an oligopyrimidine:oligopurine sequence with a single stranded TFO is reminiscent in many aspects of the formation of double-helical structures by association of two Watson-Crick complementary single strands. It is accepted nucleation-zipping model for formation both double helix and triple helix molecules, but association and dissociation rate constants of triple helices are 100 or more times slower than those of double helices.

\section{Major limitations in practical use of TFOs}

Desirable characteristics for TFO are high sequence specificity (sequence of 17 basis is unique in human genome), high binding affinity, bioavailability, efficient cellular uptake and resistance to degradation by cellular nucleases. TFO can by modified in several ways; these modifications can affect affinity and specificity of TFO molecule to duplex, improve the degradation resistance or to provide TFO molecule by additional functionality (e.g. by attaching of DNA-damaging agent).

The major drawback of G-rich (polypurine) TFO is the tendency, at physiologic potassium concentrations, to selfassociate into quartets and form tetrads, which inhibit triplex formation $(1,10)$. Nevertheless, this drawback can be overcome by chemical modifications, to be specific by the amidation of the oligonucleotide during its synthesis or by converting of phosphodiester bonds in a TFO to positively-charged linkages (moreover, this modification can increase the formation of triplex DNA dramatically) (9). Chemical modification can include every internucleoside linkage, as there is no need for an enzyme like RNase H to recognize and act upon the triplex. For most modifications of purine triplex formation is the affinity constant nearly temperature-independent. In contrast, the pyrimidine triplex is strongly favored at lower temperatures (39).

The $\mathrm{pH}$ limitation of pyrimidine motif can be decreased by base modification of cytosine to 5-methylcytosine. Effect of 5-methylcytosine is due to the contribution of the methyl group in the major groove to base stacking (49), and/or the exclusion of the water molecules from the groove (66). Although cytosine has been replaced with several analogues, such as 6-keto derivate of 5-methylcytydine, pseudoisocytidine, deoxycytidine (30) and 8-oxoadenine (38), up to now, only 5-methylcytosine has been tested in 


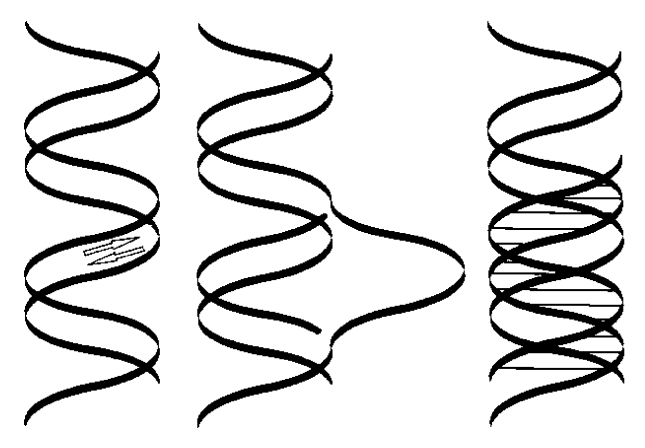

Fig. 1: DNA targeting is possible by at least are three ways - (A) by polyamides binding in the minor groove, (B) by strand displacement using PNAs (peptide nucleic acid oligomers) and (C) by triplex forming oligonucleotides binding in the major groove. (Modified from Dagle JM, Weeks DL. Oligonucleotide-based strategies to reduce gene expression. Differentiation; Research in Biological Diversity 2001;69(2-3):75-82.)

A
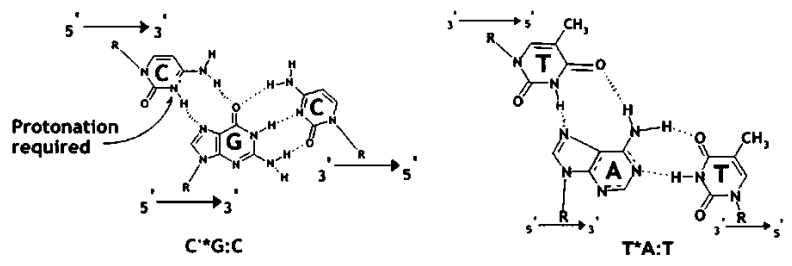

B

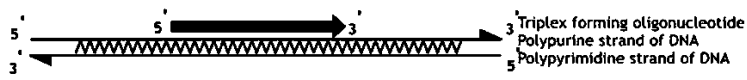

Fig. 2: Chart of the pyrimidine motif for triple helix formation. (A) Diagrams show direct Hoogsteen and WatsonCrick hydrogen bonds formed in triplets of pyrimidine motif. (B) Parallel orientation of the TFO in the pyrimidine triplex motif.

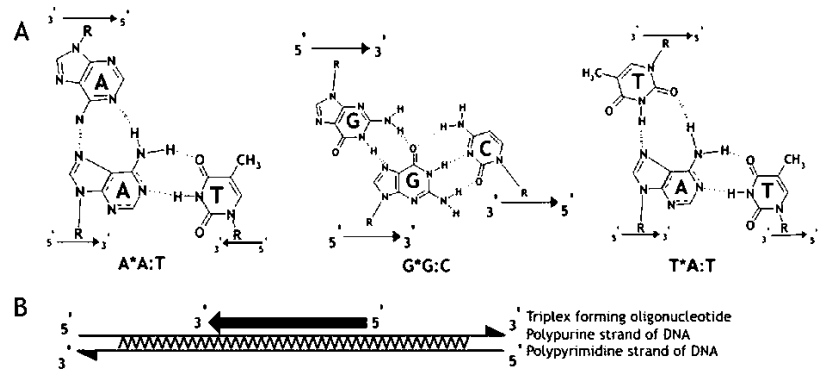

Fig. 3: Chart of the purine motif for triple helix formation. (A) Diagrams show reverse Hoogsteen and Watson-Crick hydrogen bonds formed in triplets of purine motif. (B) Antiparallel orientation of the TFO in the purine triplex motif. biological assays. Backbone modifications, such N3'->P5' phosphoramidation $(19,53,54)$ or replacement of phosphate linkage (2) improve TFO binding in vitro and stability. The application of TFO based on strands of 2'-O-Methoxy (OMe) sugar residues or 2'-O,4'-C-methylene bridged nucleic acid (2',4'-BNA) take an advantage of the fact, that RNA third strands form more stable pyrimidine motif triplexes than the corresponding DNA strands (48).

There are some major obstacles to applications of TFO under physiological conditions. Cellular uptake and delivery of TFO to target sequence into the nucleus is probably biggest obstacle for successful therapeutic utilization. Oligonucleotides are water-soluble and it may cause low efficiency in passing of cell membranes. The mechanism of cellular uptake of oligonucleotides is still poorly understood, but the conjugation of oligonucleotides with lipophilic substances to enhance membrane permeability has been proven. Triplex formation involves binding of a negatively charged third strand to a double-negatively charged duplex. Experimentally the charge repulsion is neutralized by levels of $\mathrm{Mg}^{2+}$ (5-10 mM) that are much higher than in cells (43). Triplex formation involves conformational changes on the part of the TFO and partial distortion of the underlying duplex. The cytosine protonation in the pyrimidine motif triplexes is necessary for the proper formation of the second Hoogsteen hydrogen bond. Adjacent cytosines within pyrimidine TFO often destabilize triplex, compare to TFO with isolated cytosines (65). Traditionally this has been ascribe to charge-charge repulsion effects (60), but recently incomplete protonation of adjacent cytosines was suggested to be the critical factor (51).

Aside from issues of cellular uptake, function of $\mathrm{C}^{+} / \mathrm{GC}$ TFO at physiological $\mathrm{pH}$ and salt concentration or number of related binding sites is the problem of accessibility of DNA sequence for binding of TFO. Access to a site can be lost if DNA binding protein already occupies the major groove or if the target sequence is covered by a nucleosome. It is clear that in vivo at least some sites must be free for TFO access, but mere presence of a purine-rich stretch on one strand of the DNA duplex does not guarantee triplex formation.

\section{Applications of TFO}

Postel E.H. and colleagues (46) demonstrated the first example of TFO-directed inhibition of gene transcription in cells on the human c-myc oncogene. The effects of TFOs on transcription is being studied in many cells and chromosomal targets including, integrated HIV, genes for c-myc, HER2/neu, the interlukin-2 receptor, the epidermal growth factor receptor, aldehyde dehydrogenase etc $(45,59)$. In general, these studies have shown a repression of mRNA or protein produced from the targeted gene by as much as $50-90 \%$. It is possible to link TFOs to polyamines and take an advantage of multiple effects of these molecules. In one specific case, TFO's complexed with polyamines increased 
the stability of the triplex and decreased the c-myc mRNA levels in MCF-7 breast cancer cells by $50-65 \%$ (52).

The triplex-forming potential of TFOs measured in vitro does not necessarily correlate with the ability of TFOs to affect expression of a targeted gene in vivo, but bioactivity implies at least some degree of target accessibility. It has been shown that targeting efficiency is sensitive to the biology of the cell, specifically, cell cycle status. Targeted mutagenesis was variable across the cycle with the greatest activity in $\mathrm{S}$ phase (35).

\section{Conclusion}

The use of oligonucleotides to control gene expression has long fascinated researchers because of the potential to rapidly generate potent and specific agents. This review covers only part of oligonucleotide-based approaches, but it shows number of very attractive features of this methods, suggesting that TFOs can be used to regulate gene expression or to mediate site-specific genome modification. This capacity is based on the ability of the TFOs to form triplexes with sequence specificity, to provoke action of DNA repair machinery. Potential recombination and gene correction is next step in this cascade. Although an effort is still necessary on the field of development of TFOs with robust gene targeting activity and highly efficient way of delivery TFOs into the nucleus, recent data suggest that this effort will be rewarded. We have studied TFOs features on eukaryotic cells stably expressing luciferase gene. We added to the luciferase gene short sequence recognized by TFO, located either in regulatory or in coding sequence of luciferase gene. We have demonstrated that this approach can be used during development of new drugs for gene repair. Our results will be published elsewhere.

\section{Acknowledgement}

I would like to thank Prof. Miroslav Červinka, Head of the Department, for helpful discussions and suggestions.

\section{References}

1. Arimondo PB, Garestier T, Helene C, Sun JS. Detection of competing DNA structures by thermal gradient gel electrophoresis: from self-association to triple helix formation by $(\mathrm{G}, \mathrm{A})$-containing oligonucleotides. Nucleic Acids Res 2001;29(4):E15

2. Arya DP, Bruice TC. Triple-helix formation of DNA oligomers with methylthiourea-linked nucleosides (DNmt): a kinetic and thermodynamic analysis. Proc Natl Acad Sci U S A 1999;96(8):4384-9.

3. Bates PJ, Macaulay VM, McLean MJ et al. Characteristics of triplex-directed photoadduct formation by psoralen-linked oligodeoxynucleotides. Nucleic Acid Res 1995;23(21):4283-9.

4. Behe MJ. An overabundance of long oligopurine tracts occurs in the genome of simple and complex eukaryotes. Nucleic Acids Res 1995;23(4):689-95.

5. Biet E, Maurisse R, Dutreix M, Sun_JS. Stimulation of RecA-mediated D-loop formation by oligonucleotide-directed triple-helix formation: guided homologous recombination (GOREC). Biochemistry 2001:40(6):1779-86.

6. Blume SW, Lebowitz J, Zacharias $\mathrm{W}$ et al. The integral divalent cation within the intermolecular purine* purine. pyrimidine structure: a variable determinant of the potential for and characteristics of the triple helical association. Nucleic Acids Res 1999;27(2):695-702.

7. Braasch DA, Corey DR. Novel antisense and peptide nucleic acid strategies for controlling gene expression. Biochemistry 2002;41(14):4503-10.
8. Cooney M, Czernuszewicz G, Postel EH, Flint SJ, Hogan ME. Site-specific oligonucleotide binding represses transcription of the human c-myc gene in vitro. Science 1988;241(4864):456-9

9. Dagle JM, Weeks DL. Positively charged oligonucleotides overcome potassiummediated inhibition of triplex DNA formation. Nucleic Acids Res 1996;24(11) 2143-9.

10. Dagle JM, Weeks DL. Oligonucleotide-based strategies to reduce gene expression. Differentiation 2001;69(2-3):75-82.

11. Datta HJ, Chan PP, Vasquez KM, Gupta RC, Glazer PM. Triplex-induced recombination in human cell-free extracts. Dependence on XPA and HsRad51. J Biol Chem 2001;276(21):18018-23.

12. Demidov VV, Potaman VN, Frank_Kamenetskii MD et al. Stability of peptide nucleic acids in human serum and cellular extracts. Biochem Pharmacol 1994; 48(6):1310-3.

13. Dervan PB, Burli RW. Sequence-specific DNA recognition by polyamides. Curr Opin Chem Biol 1999;3(6):688-93.

14. Durland RH, Rao TS, Bodepudi V, Seth DM, Jayaraman K, Revankar GR. Azole substituted oligonucleotides promote antiparallel triplex formation at non-homopurine duplex targets. Nucleic Acids Res 1995;23(4):647-53.

15. Duval-Valentin G, Takasugi M, Helene C, Sage E. Triple helix-directed psoralen crosslinks are recognized by Uvr(A)BC excinuclease. J Mol Biol 1998;278(4): 815-25.

16. Duval-Valentin G, Thuong NT, Helene C. Specific inhibition of transcription by triple helix-forming oligonucleotides. Proc Natl Acad Sci U S A 1992;89(2) 504-8.

17. Ebbinghaus SW, Gee JE, Rodu B, Mayfield CA, Sanders G, Miller DM. Triplex formation inhibits HER-2/neu transcription in vitro. J Clin Invest 1993;92(5): 2433-9

18. Egholm M, Buchardt O, Christensen L et al. PNA hybridizes to complementary oligonucleotides obeying the Watson-Crick hydrogen-bonding rules. Nature 1993; 365(6446):566-8

19. Escude C, Giovannangeli C, Sun JS et al. Stable triple helices formed by oligonucleotide N3' $\rightarrow$ P5' phosphoramidates inhibit transcription elongation. Proc Natl Acad Sci U S A 1996;93(9):4365-9.

20. Faruqi AF, Datta HJ, Carroll D, Seidman MM, Glazer PM. Triple-helix formation induces recombination in mammalian cells via a nucleotide excision repair-dependent pathway. Mol Cell Biol 2000;20(3):990-1000.

21. Faruqi AF, Seidman MM, Segal DJ, Carroll D, Glazer PM. Recombination induced by triple-helix-targeted DNA damage in mammalian cells. Mol Cell Biol 1996;16(12):6820-8.

22. Felsenfeld G. DDR aRA. Formation of a three-stranded poly-nucleotide molecule. J Am Chem Soc 1957:79:2023-4.

23. Francois JC, Saison_Behmoaras T, Helene C. Sequence-specific recognition of the major groove of DNA by oligodeoxynucleotides via triple helix formation. Footprinting studies. Nucleic Acids Res 1988;16(24):11431-40.

24. Gambari R. Peptide-nucleic acids (PNAs): a tool for the development of gene expression modifiers. Curr Pharm Des 2001;7(17):1839-62.

25. Gee JE, Blume S, Snyder RC, Ray R, Miller DM. Triplex formation prevents Sp1 binding to the dihydrofolate reductase promoter. J Biol Chem 1992;267(16): 11163-7.

26. Havre PA, Glazer PM. Targeted mutagenesis of simian virus 40 DNA mediated by a triple helix-forming oligonucleotide. J Virol 1993;67(12):7324-31.

27. Havre PA, Gunther EJ, Gasparro FP, Glazer PM. Targeted mutagenesis of DNA using triple helix-forming oligonucleotides linked to psoralen. Proc Natl Acad Sci U S A 1993;90(16):7879-83.

28. Helene C. The anti-gene strategy: control of gene expression by triplex-formingoligonucleotides. Anticancer Drug Des 1991;6(6):569-84.

29. Hoogsteen. The structure of crystals containing a hydrogen-bonded complex of 1 methylthymine and 9-methyladenine. Acta Crystallograf 1959;12:822).

30. Huang CY, Bi G, Miller PS. Triplex formation by oligonucleotides containing novel deoxycytidine derivatives. Nucleic Acids Res 1996;24(13):2606-13.

31. Chan PP, Lin M, Faruqi AF, Powell J, Seidman MM, Glazer PM. Targeted correction of an episomal gene in mammalian cells by a short DNA fragment tethered to a triplex-forming oligonucleotide. J Biol Chem 1999;274(17):11541-8.

32. Cho J, Parks ME, Dervan PB. Cyclic polyamides for recognition in the minor groove of DNA. Proc Natl Acad Sci U S A 1995;92(22):10389-92.

33. Kuhn H, Demidov VV, Nielsen PE, Frank_Kamenetskii MD. An experimental study of mechanism and specificity of peptide nucleic acid (PNA) binding to duplex DNA. J Mol Biol 1999;286(5):1337-45.

34. Luo Z, Macris MA, Faruqi AF, Glazer PM. High-frequency intrachromosomal gene conversion induced by triplex-forming oligonucleotides microinjected into mouse cells. Proc Natl Acad Sci U S A 2000;97(16):9003-8.

35. Majumdar A, Puri N, Cuenoud B et al. Cell cycle modulation of gene targeting by a triple helix-forming oligonucleotide. J Biol Chem 2003;278(13):11072-7.

36. Mayfield C, Ebbinghaus S, Gee J et al. Triplex formation by the human Ha-ras promoter inhibits Sp1 binding and in vitro transcription. J Biol Chem 1994; 269(27):18232-8.

37. Mergny JL, Sun JS, Rougee M et al. Sequence specificity in triple-helix formation: experimental and theoretical studies of the effect of mismatches on triplex stability. Biochemistry 1991;30(40):9791-8. 
38. Miller PS, Bi G, Kipp SA, Fok V, DeLong RK. Triplex formation by a psoralenconjugated oligodeoxyribonucleotide containing the base analog 8-oxo-adenine. Nucleic Acids Res 1996;24(4):730-6.

39. Mills M, Arimondo PB, Lacroix L, Garestier T, Klump H, Mergny JL. Chemica modification of the third strand: differential effects on purine and pyrimidine triple helix formation. Biochemistry 2002;41(1):357-66.

40. Nielsen PE. Peptide nucleic acids as therapeutic agents. Curr Opin Struct Biol 1999;9(3):353-7.

41. Nielsen PE. Targeting double stranded DNA with peptide nucleic acid (PNA) Curr Med Chem 2001;8(5):545-50.

42. Panyutin IG, Neumann RD. Sequence-specific DNA double-strand breaks induced by triplex forming $125 \mathrm{I}$ labeled oligonucleotides. Nucleic Acids Res 1994 22(23):4979-82

43. Pesco J, Salmon JM, Vigo J, Viallet P. Mag-indol affinity for $\mathrm{Ca}(2+)$, compartmentalization and binding to proteins: the challenge of measuring $\mathrm{Mg}(2+)$ concentrations in living cells. Anal Biochem XXXX;290(2):221-31

44. Plum G. Thermodynamics of oligonucleotide triple helices. Nucleic Acid Sciences 1997(44):241-256.

45. Postel EH. Modulation of c-myc transcription by triple helix formation. Ann N Y Acad Sci 1992;660:57-63.

46. Postel EH, Flint SJ, Kessler DJ, Hogan ME. Evidence that a triplex-forming oligodeoxyribonucleotide binds to the c-myc promoter in HeLa cells, thereby reducing c-myc mRNA levels. Proc Natl Acad Sci U S A 1991;88(18):8227-31.

47. Rapozzi V, Cogoi S, Spessotto P et al. Antigene effect in K562 cells of a PEGconjugated triplex-forming oligonucleotide targeted to the bcr/abl oncogene. Biochemistry 2002;41(2):502-10.

48. Roberts RW, Crothers DM. Stability and properties of double and triple helices: dramatic effects of RNA or DNA backbone composition. Science 1992; 258(5087):1463-6.

49. Singleton SF, Dervan PB. Influence of $\mathrm{pH}$ on the equilibrium association constants for oligodeoxyribonucleotide-directed triple helix formation at single DNA sites. Biochemistry 1992;31(45):10995-1003

50. Smulevitch SV, Simmons CG, Norton JC, Wise TW, Corey DR. Enhancement of strand invasion by oligonucleotides through manipulation of backbone charge Nat Biotechnol 1996;14(13):1700-4.

51. Sugimoto N, Wu P, Hara H, Kawamoto Y. pH and cation effects on the proper ties of parallel pyrimidine motif DNA triplexes. Biochemistry 2001;40(31): 9396-405.

52. Thomas RM, Thomas T, Wada M, Sigal LH, Shirahata A, Thomas TJ. Facilitation of the cellular uptake of a triplex-forming oligonucleotide by novel polyamine analogues: structure-activity relationships. Biochemistry 1999;38(40): 13328-37.

53. Torigoe $\mathrm{H}$. Thermodynamic and kinetic effects of N3'>P5' phosphoramidate modification on pyrimidine motif triplex DNA formation. Biochemistry 2001;40(4):1063-9.

54. Torigoe H, Hari Y, Sekiguchi M, Obika S, Imanishi T. 2'-O,4'-C-methylene bridged nucleic acid modification promotes pyrimidine motif triplex DNA formation at physiological pH: thermodynamic and kinetic studies. J Biol Chem 2001; 276(4):2354-60
55. Vasquez KM, Dagle JM, Weeks DL, Glazer PM. Chromosome targeting at short polypurine sites by cationic triplex-forming oligonucleotides. J Biol Chem 2001; 276(42):38536-41

56. Vasquez KM, Narayanan L, Glazer PM. Specific mutations induced by triplexforming oligonucleotides in mice. Science 2000;290(5491):530-3.

57. Vasquez KM, Wang G, Havre PA, Glazer PM. Chromosomal mutations induced by triplex-forming oligonucleotides in mammalian cells. Nucleic Acids Res 1999; 27(4):1176-81.

58. Vasquez KM, Wensel TG, Hogan ME, Wilson JH. High-efficiency triple-helix-mediated photo-cross-linking at a targeted site within a selectable mammalian gene. Biochemistry 1996;35(33):10712-9.

59. Vasquez KM, Wilson JH. Triplex-directed modification of genes and gene activity. Trends Biochem Sci 1998;23(1):4-9.

60. Volker J, Klump HH. Electrostatic effects in DNA triple helices. Biochemistry 1994;33(45):13502-8.

61. Wang E, Malek S, Feigon J. Structure of a G.T.A triplet in an intramolecular DNA triplex. Biochemistry 1992;31(20):4838-46.

62. Wang G, Seidman MM, Glazer PM. Mutagenesis in mammalian cells induced by triple helix formation and transcription-coupled repair. Science 1996;271(5250): $802-5$

63. Wemmer DE, Dervan PB. Targeting the minor groove of DNA. Curr Opin Struct Biol 1997;7(3):355-61.

64. White S, Szewczyk JW, Turner JM, Baird EE, Dervan PB. Recognition of the four Watson-Crick base pairs in the DNA minor groove by synthetic ligands. Nature 1998;391(6666):468-71.

65. Xodo LE, Manzini G, Quadrifoglio F. Formation of stable DNA triple helices within the human ber promoter at a critical oligopurine target interrupted in the middle by two adjacent pyrimidines. Antisense Nucleic Acid Drug Dev 1998; $8(6): 477-88$

66. Xodo LE, Manzini G, Quadrifoglio F, van der Marel GA, van Boom JH. Effect of 5-methylcytosine on the stability of triple-stranded DNA-a thermodynamic study. Nucleic Acids Res 1991;19(20):5625-31.

Submitted March 2004.

Accepted May 2004.

Mgr. Martin Mojžĭšek,

Charles University in Prague,

Faculty of Medicine in Hradec Králové,

Department of Medical Biology and Genetics, Šimkova 870, 50038 Hradec Králové,

Czech Republic. e-mail:mojzisekm@lfhk.cuni.cz 